\title{
Das Fasziendistorsionsmodell (nach Typaldos): Die 6 Störungen und deren Behandlung
}

\author{
Raimond Igel
}

Stephen Typaldos, Notfallmediziner und Osteopath, entwickelte das Fasziendistorsionsmodell (FDM). Es ist ein anatomisches Modell, das sich durch seine einfache Systematik auszeichnet und Verletzungen oder andere Krankheitsbilder auf eine oder mehrere von 6 spezifischen „Distorsionen“ der Faszien zurückführt. Für die Diagnose wesentlich ist dabei die Körpersprache des Patienten im Rahmen seiner Schilderung der Beschwerden beigemessen.

\section{Befunderhebung}

Bei der Befundung im Rahmen des FDM, die sich im Wesentlichen auf die 3 Säulen Anamnese, körperliche Untersuchung und Körpersprache stützt, ist der Patient meist selbst in der Lage, genau zu zeigen, wo seine Problematik liegt - vorausgesetzt, der Therapeut kann mit entsprechend geschultem und aufmerksamen Blick diesen körpersprachlichen Ausdruck entsprechend interpretieren.

So entdeckte 1991 Typaldos das erste Triggerband. Ihm fiel auf, dass seine Patienten immer wieder die gleiche Körpersprache benutzten. Er entwickelte das FDM, in dem er Klinik und Körpersprache des Patienten zu einer Diagnose zusammenfasste und eine darauf abgestimmte Therapie definierte. Auf diese Weise entdeckte er 6 verschiedene mögliche Folgen von Verletzungen, die sich als Störungen innerhalb der Faszien des Bewegungsapparats manifestieren können:

1. Triggerband

2. Kontinuumdistorsion

3. hernierter Triggerpunkt

4. Zylinderdistorsion

5. Faltdistorsion

6. tektonische Fixation

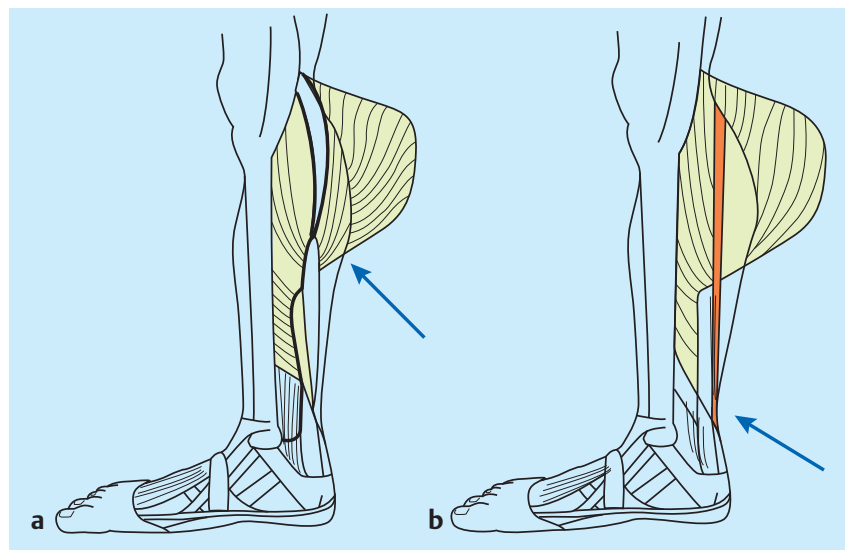

Abb. 1 a und $\mathbf{b}$ a Verdrehtes Triggerband. b Behandeltes Triggerband. Quelle: modifiziert nach [1]

\section{Triggerband}

Eine Überbelastung im Gewebe, der die Faszien unter Umständen nicht standhalten können, führt zu einer Distorsion (= „Verdrehung“ im Kontext des FDM). Da die Fasern der Faszien parallel zum Muskel verlaufen, können Scherkräfte hier zu Verletzungen führen. Dadurch kommt es zu Verdrehungen oder Aufspaltungen der Faszien.

Im FDM werden 3 Störungsformen bei Triggerbändern unterschieden:

1. Verdrehtes Faszienband ( Ein verdrehtes Faszienband gleicht einem Schal, dessen Anfang und Ende fixiert sind und der in der Mitte verdreht wird.

2. Aufspaltung einer Faszie ( $\triangleright$ Abb.2): Durch das Aufspalten der Fasern kommt es zu einer Verkürzung der Faszien. Dies erklärt die auftretenden Bewegungseinschränkungen in der Achse des Triggerbands. Aufgespaltene Triggerbänder können chronisch werden. Bei einer gestörten Wundheilung kommt es zu einer Verklebung von Geweben. Die sog. Crosslinks verursachen weitere Einschränkungen der Beweglichkeit. Dies führt wiederum zum Auftreten von neuen Triggerbändern.
3. Einlagerung von Kalzium entlang einer Stresslinie: Wirkt ein permanenter Zug auf die Faszie (z. B. auf die Achillessehne beim Langstreckenläufer), kann eine „Stresslinie“ entstehen. Der Körper reagiert mit Einlagerung von Kalziumkristallen entlang der Faszie. Sie bewirken eine „Versteifung“ im Verlauf dieser Linie. Die Faszie verliert dadurch in erheblichem Maße ihre Geschmeidigkeit.

Dies führt dazu, dass es bei extremen Bewegungen, die der Faszie mehr Flexibilität abverlangen, zu Schmerzen kommt. Stresslinien entstehen über einen langen Zeitraum. Sie können nur durch intensive Bewegung bzw. Mobilisierung gebessert werden.

\section{Körpersprache}

Der Patient streicht mit den Fingern entlang einer Linie und zeigt so den Verlauf des Triggerbands in der Faszie ( $\triangleright$ Abb. $\mathbf{3}$ ). Meist wird ein ziehender, brennender Schmerz entlang der gezeigten Linie verbalisiert. Die Stärke des Druckes, der beim Zeigen auf die Körperstelle angewandt wird, kann Hinweise geben, wie tief das betroffene Triggerband liegt. 


\section{Untersuchung}

- Eine verringerte Beweglichkeit lässt auf eine Verkürzung der Faszien schließen.

- Eine Schmerzunahme bei passiven oder aktiven Bewegungstests deutet auf eine Distorsion hin.

- Palpatorisch ist eine fühlbare Verhärtung bzw. Verklebung der betroffenen Faszie in der Tiefe zu spüren.

- Typisch für chronische Triggerbänder ist ein Anlaufschmerz. Dieser tritt anfänglich nach längerer Ruhigstellung (z.B. längerem Sitzen) auf. In der Ruhephase kommt es zur Bildung von Adhäsionen, die sich unter Bewegung wieder lösen können. Dies passiert so lange, bis das Triggerband komplett verheilt ist oder behandelt wurde.

\section{Behandlung}

Ziel einer Triggerbandbehandlung sind die Korrektur der Verdrehung, die Auflösung der Adhäsion und der Verschluss der Faszie. Beim kalzifizierten Triggerband gilt es, die Kalzifizierung aufzulösen.

Die Behandlung eines Triggerbands erfolgt mit der Daumentechnik. Mit angemessen hohem Druck folgt der Therapeut mit dem Daumen der Distorsion bis zum knöchernen Ende. Anschließende Eisabreibungen im behandelten Bereich wirken schmerzlindernd und beugen Hautreizungen vor.

\section{Kontinuumdistorsion}

Im Hochleistungssport (aber auch im Alltag) kommt es häufig zur Überlastung des muskuloskelettalen Systems. Folge sind Störungen der Übergangszone zwischen Faszie und Knochen, sog. Kontinuumdistorsionen (Continuumdistorsion, CD). Es kommt zu einer Veränderung der Matrix von Faszie oder Knochen. Neben den auftretenden Schmerzen kommt es auch zu mangelnden sensorischen Informationsübertragungen. Typische Beispiele dafür sind Tennis- und Golferellenbogen.

Es werden 2 Formen der Kontinuumdistorsion unterschieden:

- invertierte CD: die Faszie ragt in den Knochen hinein

- evertierte CD: die Knochenmatrix ragt in die Faszie

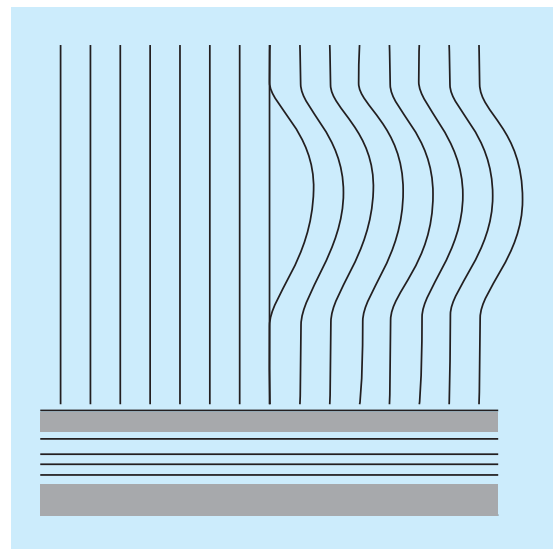

- Abb.2 Aufgespaltenes Triggerband. Quelle: modifiziert nach [1]

\section{Körpersprache}

Der Patient zeigt meist mit einer Fingerspitze auf einen bestimmten Punkt, der sich in der Nähe eines Gelenks befindet. Des Weiteren berichten die meisten Patienten über einen stechenden, punktuellen Schmerz in diesem Bereich.

\section{Untersuchung}

Die Palpation ist die einzige Möglichkeit, zwischen einer evertierten oder invertierten $\mathrm{CD}$ zu unterscheiden:

- Bei der evertierten CD ist eine „reiskorngroße“ Veränderung (Knochenvorsprung) zu spüren.

- Bei der invertierten CD fühlt es sich wie eine kleine Delle am Knochen an.

\section{Behandlung}

Um eine Kontinuumdistorsion optimal zu behandeln, ist es wichtig, die Störungen der Übergangszone zu finden. Durch hohen Daumendruck auf den Schmerzpunkt wird die Übergangszone dazu gebracht, wieder in eine neutrale Konfiguration zu wechseln. Unter dem Daumen des Therapeuten ist deutlich zu spüren, wie das „Reiskorn“ langsam aufweicht. Dabei gilt das Prinzip „Alles oder Nichts“, d.h. der Therapeut bleibt so lange auf der CD, bis die Übergangszone normalisiert ist und der Patient keine Schmerzen mehr unter dem Daumendruck spürt.

$\mathrm{Zu}$ beachten ist, dass nach einer evertierten CD-Behandlung 24 Stunden keine Manipulationsthrusts in diesem Bereich erfolgen sollten, damit sich die vorgenommene Korrektur festigen kann. Andernfalls besteht das Risiko, die soeben behobene Störung wieder „herauszuziehen“. Bei der invertierten $\mathrm{CD}$ ist hingegen eine Manipu-

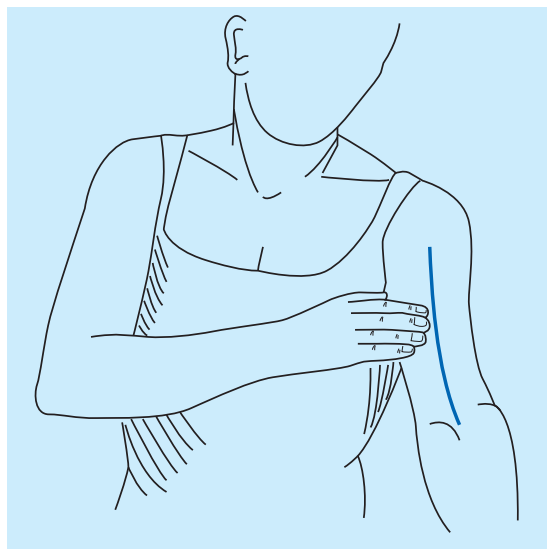

- Abb. 3 Körpersprache des Patienten bei einem Triggerband. Quelle: modifiziert nach [1]

lation günstig, da durch Thrusts der Bereich, der sich in den Knochen verlagert hat, wieder herausgezogen und eine neutrale Konfiguration erzeugt wird.

\section{Hernierter Triggerpunkt (HTP)}

Unter einem HTP versteht man eine Vorwölbung (Protrusion) von Gewebe, die aus einer tieferen Gewebeschicht durch eine darüberliegende Faszienschicht dringt. Trotz der Bezeichnung „Triggerpunkt" entspricht ein HTP nicht den bekannten Triggerpunkten eines Muskels.

Ein hernierter Triggerpunkt entsteht durch hohe Druckunterschiede im Thoraxbereich bei gleichzeitiger Kraftanstrengung unter Bewegung. Dies führt zu einer Öffnung der Faszienschichten. Das Gewebe gibt dann dem erhöhten Druck nach und drückt sich durch die entstandene Öffnung nach außen. Beim Nachlassen des Druckes wird die Protrusion „eingeklemmt“. Es kommt zu schmerzhaften Bewegungseinschränkungen in der betroffenen Region.

Vor allem im Hochleistungssport, insbesondere beim Krafttraining, wo extreme Kraftanstrengungen erbracht werden müssen, kommen HTPs oft vor. Das Beschwerdebild des Athleten reicht von Hexenschuss oder Kopfschmerzen bis zu schmerzfreien Einschränkungen der Beweglichkeit. Ursache dafür ist die durch den HTP entstandene erhöhte Spannung der Faszien sowohl in seinem direkten als auch weit entferntem Umfeld. Durch Vernetzungen der Faszien im ganzen Körper kann diese Spannung somit auch auf andere Körperbereiche übertragen werden. 


\section{Körpersprache}

Der Patient drückt mit mehreren Fingern, mit dem Daumen oder der Faust auf das Schmerzareal. Gelegentlich drückt er fest in das Gewebe, um so selbst die Protrusion zu reponieren. HTPs werden oft als dumpfer, in der Tiefe liegender Schmerz beschrieben. Patienten klagen über Bewegungseinschränkungen und beschreiben die betroffene Region als „verspannt“.

\section{Untersuchung}

Sowohl die Untersuchung als auch die Behandlung erfolgen mit dem Daumen. Die schmerzhaften Gewebsprotrusionen, die in der Tiefe liegen, sind mit einer gelartigen Murmel vergleichbar. Durch den erhöhten Druck des Daumens kommt es zu ausstrahlenden Schmerzen, gelegentlich auch zu neurologischen Erscheinungen, z.B. Kribbeln im Arm, die nach der Beendigung des Druckes wieder verschwinden. Im Bereich des HTPs ist sowohl die aktive als auch passive Beweglichkeit eingeschränkt, das Gleiten der Faszienschichten ist gestört.

\section{Behandlung}

Unter großem Druck auf den HTP erfolgt die - angestrebte vollständige - Reposition des ausgetretenen Gewebes durch die „Bruchpforte“. Hierbei ist der korrekte Winkel des Druckes entscheidend. Es ist hilfreich, den Patienten so zu positionieren, dass die Bruchpforte entspannt und geöffnet ist. Sobald der Patient keinen Druckschmerz mehr spürt und der Therapeut fühlen kann, dass die Reposition erfolgreich war, wird der Druck noch für ca. 10 Sekunden gehalten.

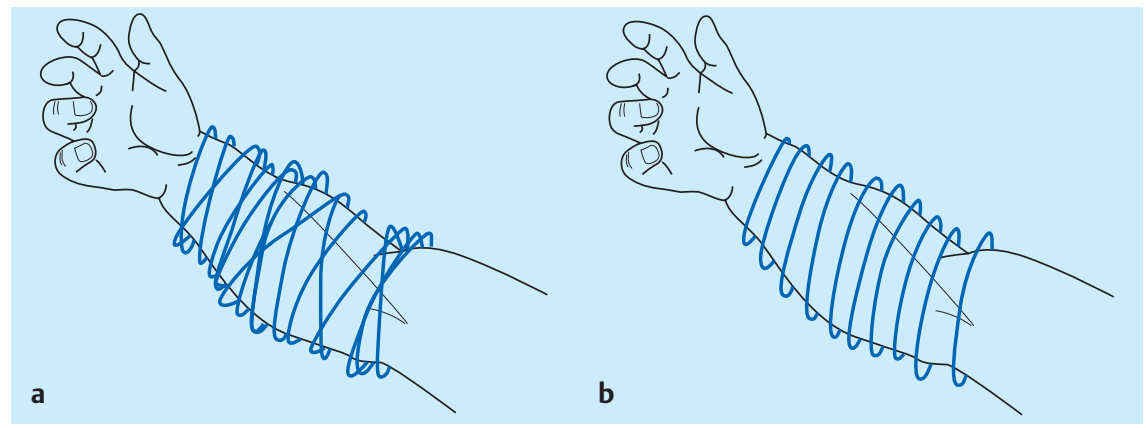

- Abb. 4a und b a Zylinderdistorsion mit „verhakten“ Spiralwindungen. b Normalzustand. Quelle: modifiziert nach [1]

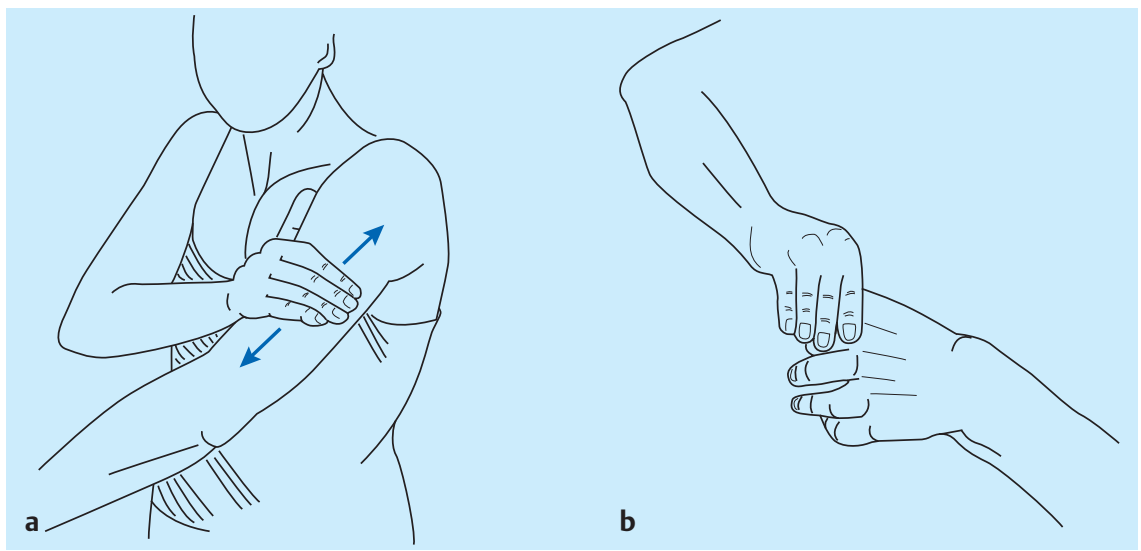

- Abb. 5 Körpersprache bei einer Zylinderdistorsion. Quelle: modifiziert nach [1]

\section{Zylinderdistorsion}

Eine Zylinderdistorsion ist das „Verhaken“ der zirkulären Spiralwindungen der oberflächlichen Faszien ( $\$$ Abb. 4). Sie tritt häufig auf. Kribbeln, Missempfindungen und Krämpfe sind typische Symptome. Eine Fehlspannung innerhalb der oberflächlichen Faszien, die Gefäße und Nerven enthalten, führt zu entsprechenden nervalen und vaskulären Störungen. Cha- rakteristisch ist, dass die damit verbundenen Schmerzen üblicherweise sehr plötzlich auftreten und ebenso ad hoc wieder Schmerzfreiheit eintritt.

\section{Körpersprache}

Die Körpersprache kann hier sehr unterschiedlich sein. Häufig streicht der Patient mit der Hand über die betroffene Region. Aufgrund der unterschiedlichen Verläufe und Symptome von Zylinderdistorsionen 


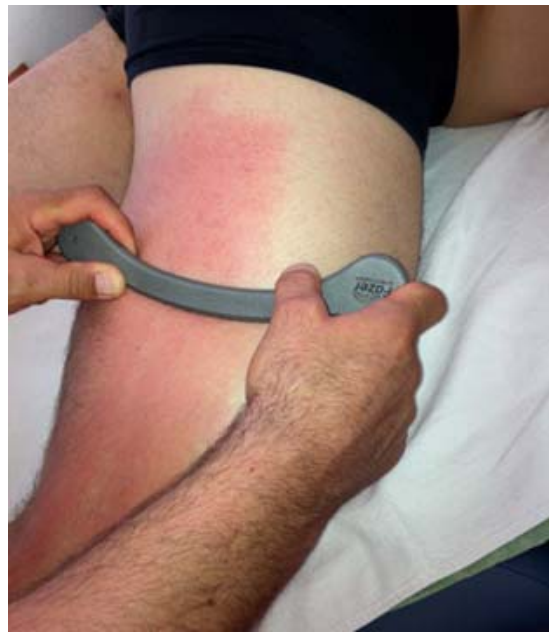

- Abb. 6 Behandlung einer Zylinderdistorsion mit einem Fazer. Quelle: (c) Raimond Igel

sind jedoch weitere körpersprachliche Ausdrucksformen möglich, wie Kneten, Kneifen, Reiben, „Handschuh ausziehen“ und Schütteln ( $\triangleright$ Abb. 5). Schwellungsgefühl, Taubheit der betroffenen Extremität und auch wiederkehrende Krämpfe ohne vorausgegangene Belastung können die Zylinderdistorsion begleiten.

\section{Untersuchung}

Zylinderdistorsionen sind palpatorisch nicht zu spüren, d. h. nicht tastbar. Lediglich durch aktive Bewegungen kann der Patient den Schmerz auslösen. Passive Bewegungen sind hingegen schmerzfrei möglich.

\section{Behandlungen}

Um die „verhakten“ Spiralwindungen zu lösen und den Zylinder in seine normale Form zurückzubringen, kann die Therapie sowohl manuell als auch unter Einsatz von Hilfsmitteln erfolgen. Sehr wirkungsvoll hat sich in der Praxis hier die geräteunterstützte Faszientherapie ( $\triangleright$ Abb. 6) erwiesen.

\section{Faltdistorsion}

Um die maximale Beweglichkeit eines Gelenkes zu gewährleisten, ist die Faszie, die das Gelenk umschließt, in Falten gelegt. Diese Falten stellen ein verschleißfreies Bewegen sicher und wirken wie ein Puffer gegen einwirkende Kräfte auf die Gelenkkapsel. Beim Speerwurf bspw. steht hier das Schultergelenk besonders im Fokus beim Abwurf des Speeres.

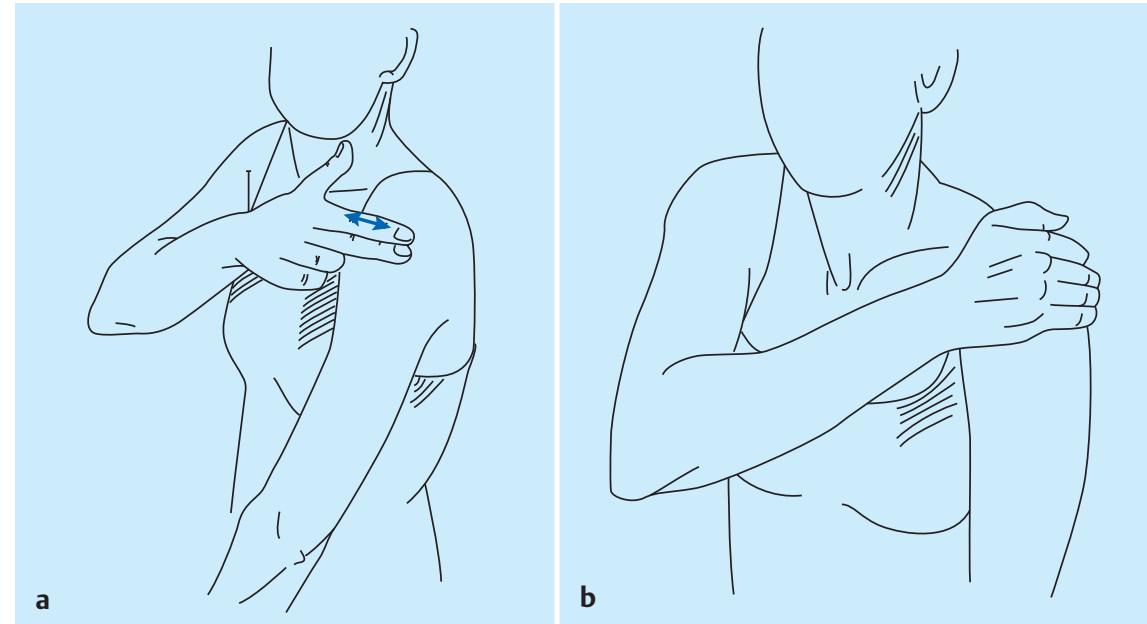

- Abb. 7 a und b Körpersprache bei Einfaltdistorsion (a) und Entfaltdistorsion (b). Quelle: mod. nach [1]

Unter einer Faltdistorsion versteht man eine Störung der Gelenkfaszie in 3 Ebenen. Es werden 2 Arten unterschieden:

- Einfaltdistorsion: Sie kann z.B. durch einen Sturz entstehen oder auch durch von außen einwirkende Kräfte (z.B. Krafttraining, Reißen). Dabei erfolgt eine direkte Wirkung auf das Gelenk, wodurch es zu einer Komprimierung der Kapsel bzw. Faszie kommt, häufig in Kombination mit einer Rotation. Bei der darauffolgenden Entfaltung der Faszie kommt es dann zu einer Verdrehung der Faszie. Die Entfaltung ist somit nicht korrekt möglich und schmerzhaft. Die Einfaltung stellt sich jedoch dann wieder als beschwerdefrei dar.

- Entfaltdistorsion: Durch einen plötzlichen Zug an der Gelenkkapsel werden die Falten zu weit auseinandergezogen. In Verbindung mit einer Rotation kommt es beim anschließenden Einfalten zur Verdrehung der Gelenkfaszien (,Zerknitterung“). Die Entfaltung stellt sich dabei beschwerdefrei dar, die Einfaltung ist jedoch schmerzhaft.

\section{Körpersprache}

Faltdistorsionen werden vom Patienten immer dynamisch angezeigt. Dieser hält das betroffene Gelenk, knetet es oder hält es unter gleichzeitiger Bewegung. Ebenso kommt es vor, dass auf mehrere Punkte um das Gelenk gezeigt wird. Bei der Entfaltdistorsion versucht er, die betroffene Extremität zu komprimieren. Bei der Einfaltdistorsion streicht er mit den Fingern quer über das Gelenk, was eine Besonderheit der Körpersprache darstellt ( $\triangleright$ Abb. 7).

\section{Untersuchung}

Um festzustellen, ob es sich um eine Einfalt- oder Entfaltdistorsion handelt, wird mittels Traktion und Kompression getestet. Bei der Traktion wird eine Einfaltdistorsion Schmerzen verursachen, die Entfaltdistorsion hingegen ist schmerzfrei. Bei der Kompression ist die Einfaltdistorsion hingegen schmerzfrei und die Entfaltdistorsion schmerzhaft. Bei beiden Faltdistorsionen verursacht eine passive endgradige Bewegung Schmerzen.

\section{Behandlung}

Ein wichtiger Aspekt für eine optimale Behandlung einer Faltdistorsion ist, dass der Therapeut möglichst Kenntnis über den genauen Unfallhergang erlangt. Diese Information ist deshalb wichtig, da der Therapeut auf diese Weise die Position des Gelenks für die Behandlung optimal einstellen kann. Durch Provokationstests findet der Therapeut die genaue Stellung heraus, in der der Schmerz am größten ist.

Bei der Einfaltdistorsion ist eine Kompressionsmanipulation, bei der Entfaltdistorsion ein Manipulationsthrust erforderlich.

\section{Tektonische Fixierung}

Unter einer tektonischen Fixierung versteht man den Verlust der Gleitfähigkeit einer Faszienfläche. Durch starken Druck oder mangelnde Bewegung kann es im Körper zum Auspressen der Flüssigkeit kommen, die sich zwischen den Faszienflächen befindet. Dies hat zur Folge, dass die Faszien 
miteinander „verkleben“, also ihre physiologische Gleitfähigkeit z.T. verlieren. Existiert eine tektonische Fixierung über einen längeren Zeitraum, kommt es durch das Fehlen der Gelenkflüssigkeit zu einer Minderversorgung des Knorpels und damit zur Degeneration der Gelenkflächen.

\section{Körpersprache}

Die Körpersprache entspricht in etwa der Faltdistorsion. Der Patient legt die Hand auf das Gelenk bzw. die Faszienfläche und hält diese fest. Ein kraftvolles Mobilisieren der betroffenen Extremität gelingt dem Patienten nicht.

\section{Untersuchung}

Die Palpation bei tektonischen Fixierungen ist unauffällig. Das Gelenk lässt sich für den Patienten schmerzfrei sowohl aktiv als auch passiv bewegen, wobei lediglich ein „hartes Gefühl“ am Ende der Bewegung zu spüren ist. Dies ist häufig der Grund, warum der Patient nicht oder erst spät einen Therapeuten aufsucht.

\section{Behandlung}

Die tektonischen Fixierungen sind die am schwierigsten zu behandelnden Distorsionen, da die Bewegungseinschränkung völlig schmerzfrei ist. Durch die Behandlung muss dafür gesorgt werden, dass sowohl die Verklebungen der Gelenkflächen, also der Faszienflächen, behoben als auch die Produktion neuer Gelenkflüssigkeit ange- regt wird, um so die Fixierung dauerhaft zu lösen.

Grundsätzlich gilt: Je kleiner das Gelenk, desto einfacher und schneller kann die fasziale Gleitfähigkeit wieder hergestellt werden. Je größer das Gelenk ist, umso größer ist in der Regel auch die Verklebung um den Bereich, der gelöst werden muss. Hier sind bekannte Techniken aus der manuellen Therapie geeignet (z.B. Translationstechniken im Kniebereich, Mobilisation Tibia über Talus).

\section{Zusammenfassung}

Genau betrachtet sind zwischen dem FDM und den Grundgedanken der Osteopathie starke Verbindungen erkennbar. Ein wichtiger Leitsatz im Rahmen von Stills Philosophie lautet: „Find it, fix it, leave it alone.“ FDM ist ein Konzept, das ebenso das Erkennen (find it) und die Behandlung (fix it) von strukturellen, bindegewebigen Störungen effizient gestaltet. Wesentlich für den Behandlungserfolg ist die konsequente Bewegung der behandelten Strukturen, um diese im Prozess der Selbstregulation (leave ist alone) zu unterstützen.

\section{Literatur}

1 Römer F. Praktisches Lehrbuch zum Fasziendistorsionsmodell. 2. Aufl. Wolfenbüttel: Römer; 2014
2 Schleip R. Lehrbuch Faszien: Grundlagen, Forschung, Behandlung. München: Urban \& Fischer/Elsevier; 2014

3 Schwind P. Faszien- und Membrantechnik. 2. Aufl. München: Urban \& Fischer/Elsevier; 2009

4 Myers TW. Anatomy Trains. Myofasziale Leitbahnen. 2. Aufl. München: Urban \& Fischer/ Elsevier; 2010

5 Typaldos S. Orthopathische Medizin. 3. Aufl. Kötzting: Wühr; 2010

6 Langer W, Hebgen E. Lehrbuch Osteopathie. Stuttgart: Haug; 2013

\section{Online}

http://dx.doi.org/10.1055/s-0034-1383009

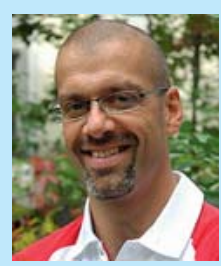

Raimond Igel Clayallee 175 14195 Berlin Ausbildung zum Krankenpfleger und Physiotherapeuten. Seit 2002 lizensierter Sportphysiotherapeut des DOSB und Mitglied des medizinischen Teams des Deutschen Leichtathletikverbands. Betreuung der Athleten bei DM, EM, WM und Olympia. 2007 Osteopathie-Examen. Spezialisierung für Faszien und Faszientherapie mit Schwerpunkt Sport und Hochleistungssport.

E-Mail: raimond.igel@web.de 